CMEARTICLE

\title{
High-grade atrioventricular block
}

Yinghao $\underline{\operatorname{Lim}}^{1}$, MBBS, MRCP, Devinder Singh${ }^{1}$, MBBS, MRCP, Kian Keong $\underline{\text { Poh }}^{1}$, FRCP, FACC

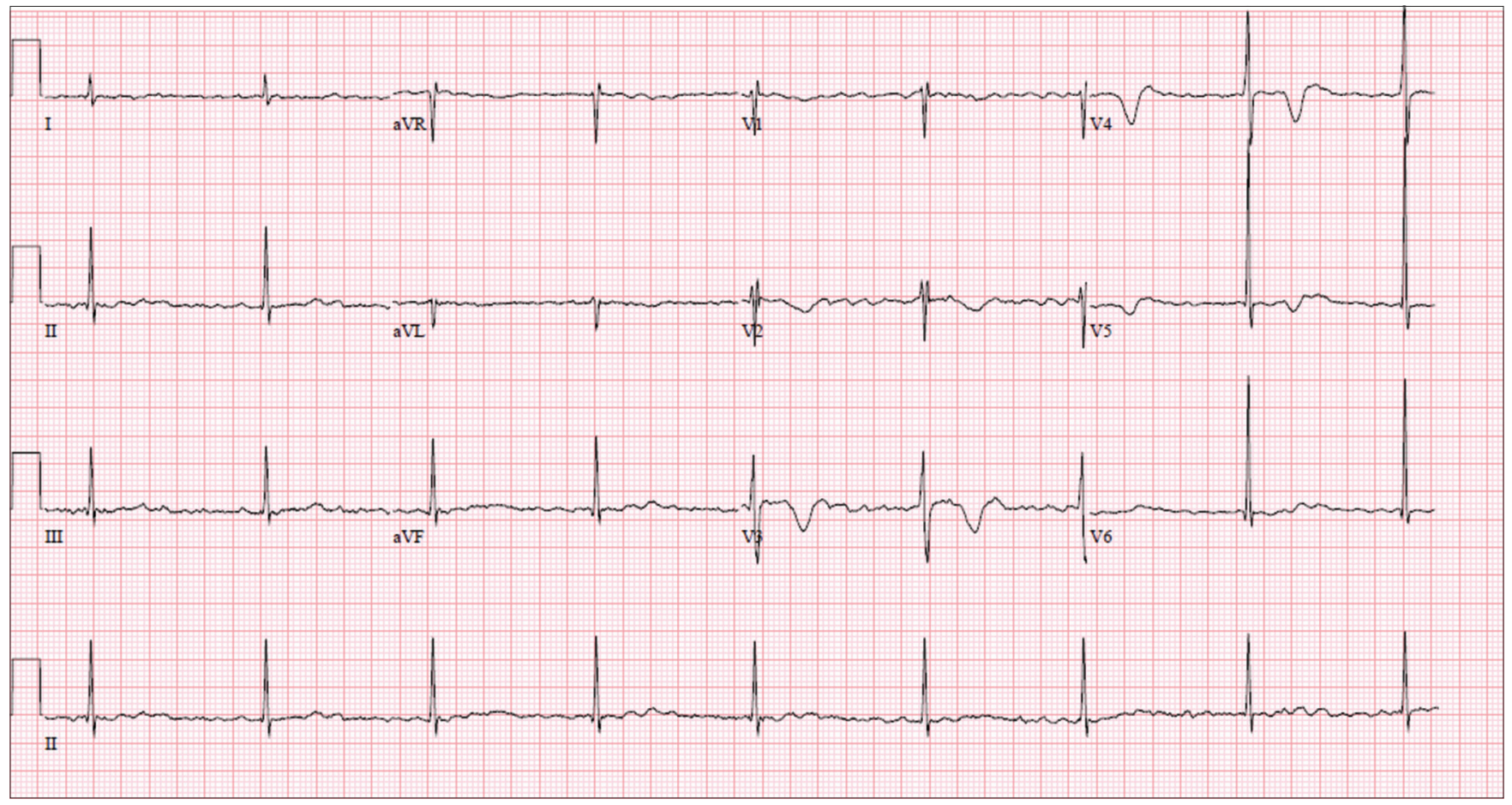

Fig. 1 Case 1: ECG performed for a patient with bradycardia during vital parameter screening.

\section{CASE 1}

\section{CLINICAL PRESENTATION}

A 66-year-old man with Stage Ilb pancreatic cancer and atrial fibrillation (AF) was admitted for chemotherapy. He was not on any rate-control drugs and was noted to be bradycardic on routine parameter monitoring. Electrocardiography (ECG) was performed (Fig. 1). What is the ECG diagnosis?

\section{ECG INTERPRETATION}

The ECG shows a normal axis and a rate of 50 beats per minute (bpm). There are no discrete, well-formed $\mathrm{P}$ waves, but a fine fibrillatory baseline is suggestive of AF (Fig. 2). QRS complexes are narrow with transition at $\mathrm{V} 3$ and $\mathrm{R}-\mathrm{R}$ intervals are regular. There are abnormal deep $T$ wave inversions in the anterior leads.

In the setting of $A F, R-R$ intervals are typically irregularly irregular. Although the patient had AF, his ECG shows an unusually low and regular heart rate without rate-control agents. This is evidence of atrioventricular (AV) dissociation consistent with complete heart block, with a narrow (supra-Hisian) escape rhythm.

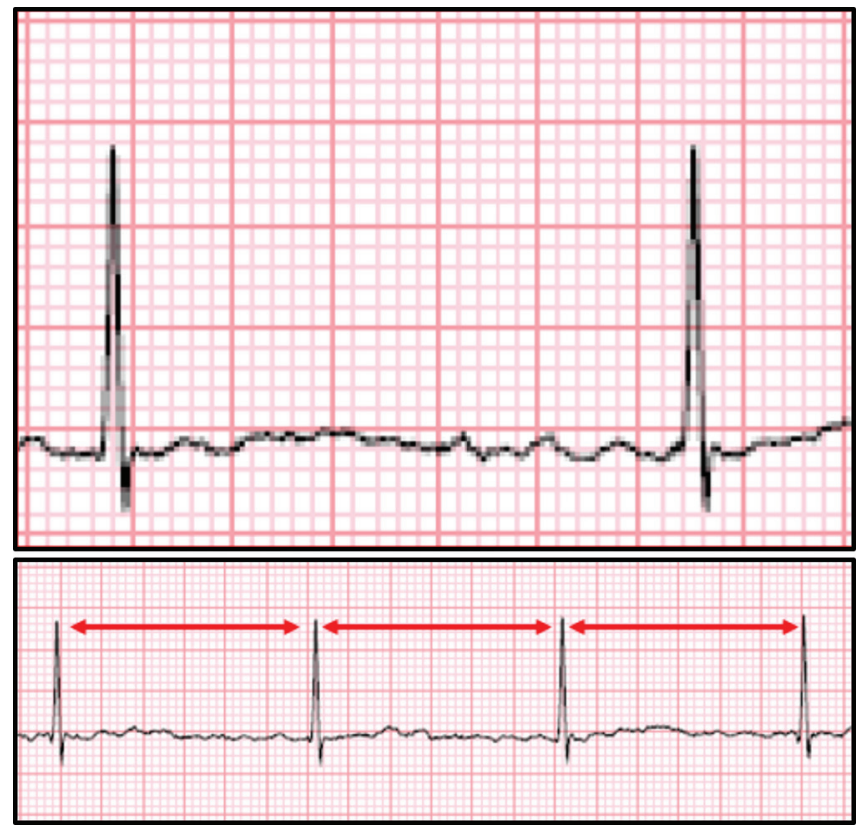

Fig. 2 Case 1: ECG shows a fine fibrillatory baseline that suggests atrial fibrillation. R-R intervals (arrows) are regular despite the atrial fibrillation. 


\section{CLINICAL COURSE}

The patient was monitored in the Coronary Care Unit (CCU), as he had transiently low resting heart rates of $35 \mathrm{bpm}$. No transvenous pacing or chronotropic agents were required, as he was haemodynamically stable and asymptomatic. Furthermore, there was no unstable or slow infra-Hisian escape rhythm. Anticoagulation treatment was discussed with the patient in view of his CHADS2VA2Sc score of 1, but was not initiated based on patient preference after weighing the risks and benefits of anticoagulation. Computed tomography coronary angiography and transthoracic echocardiography were also performed in view of his $\mathrm{T}$ wave changes but did not reveal any significant coronary artery disease or structural heart disease. A permanent pacemaker was implanted and the patient was discharged after an overnight stay.

\section{CASE 2}

\section{CLINICAL PRESENTATION}

A 78-year-old man was admitted for non-ST elevation myocardial infarction. During his admission, he was noted to be bradycardic, and an ECG was performed (Fig. 3). What is the diagnosis?

\section{ECG INTERPRETATION}

The ECG shows a normal axis and a low ventricular rate of 44 bpm. The $\mathrm{P}$ and QRS morphologies are fairly normal in appearance. There are non-conducted $P$ waves buried at the tail end of the T wave. The P-P interval is $660 \mathrm{~ms}$ (91 bpm) and R-R interval is $1,360 \mathrm{~ms}$ (44 bpm). The conducted P-R intervals are prolonged at $300 \mathrm{~ms}$. There are no ST elevations or depressions. There are two $\mathrm{P}$ waves for every conducted QRS complex. Consequently, the P-P rate is almost exactly double of the R-R rate. This is suggestive of a 2:1 AV block. Conducted QRS complexes are narrow and do not exhibit any aberrancy (Fig. 4).

\section{CLINICAL COURSE}

The patient was administered dual antiplatelet therapy and underwent percutaneous coronary intervention $(\mathrm{PCI})$ on the same day. The cause was found to be a significant mid left anterior descending artery lesion. The patient was managed in the $\mathrm{CCU}$ after $\mathrm{PCl}$, and was haemodynamically stable and asymptomatic. Despite revascularisation, he had persistent 2:1 AV block. Thus, a dual chamber permanent pacemaker was implanted. The postimplantation ECG (Fig. 5) and chest radiograph (Fig. 6) are shown.

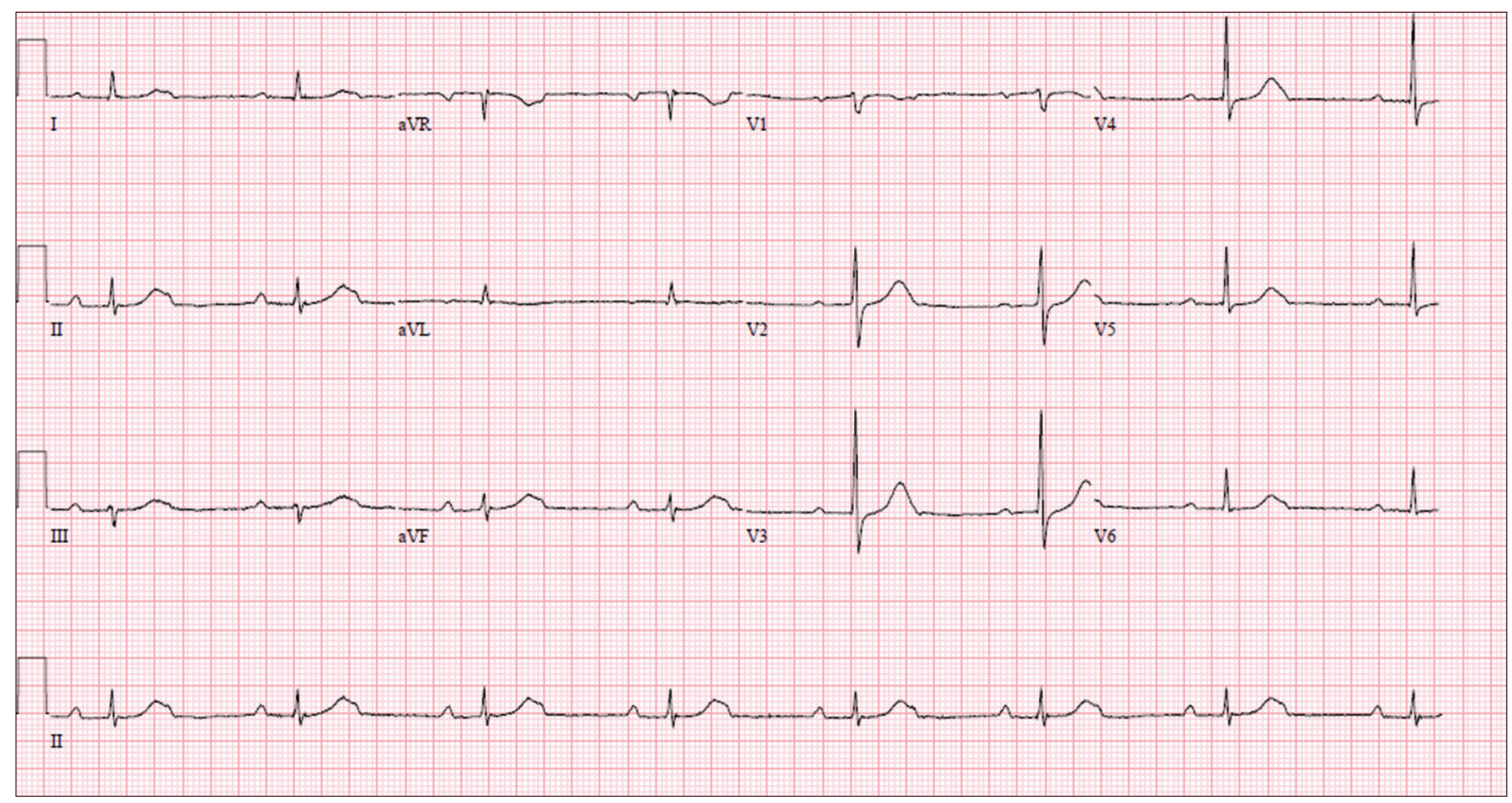

Fig. 3 Case 2: ECG performed for a patient with bradycardia.

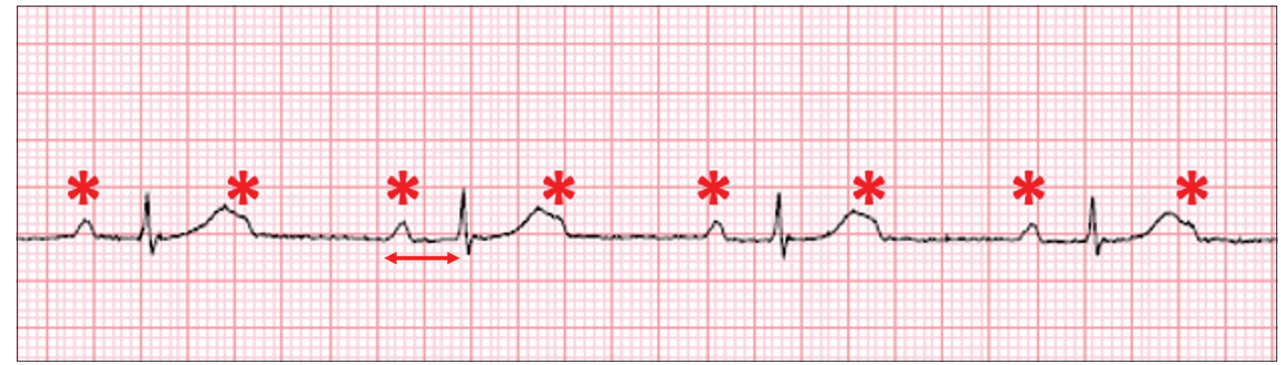

Fig. 4 Case 2: ECG shows regular P waves $\left({ }^{*}\right)$ with every other one buried at the tail end of the T wave. The P-P rate is twice that of the R-R rate. The $\mathrm{P}-\mathrm{R}$ interval for conducted beats is prolonged at $300 \mathrm{~ms}$ (arrow) and the QRS complexes are narrow. 


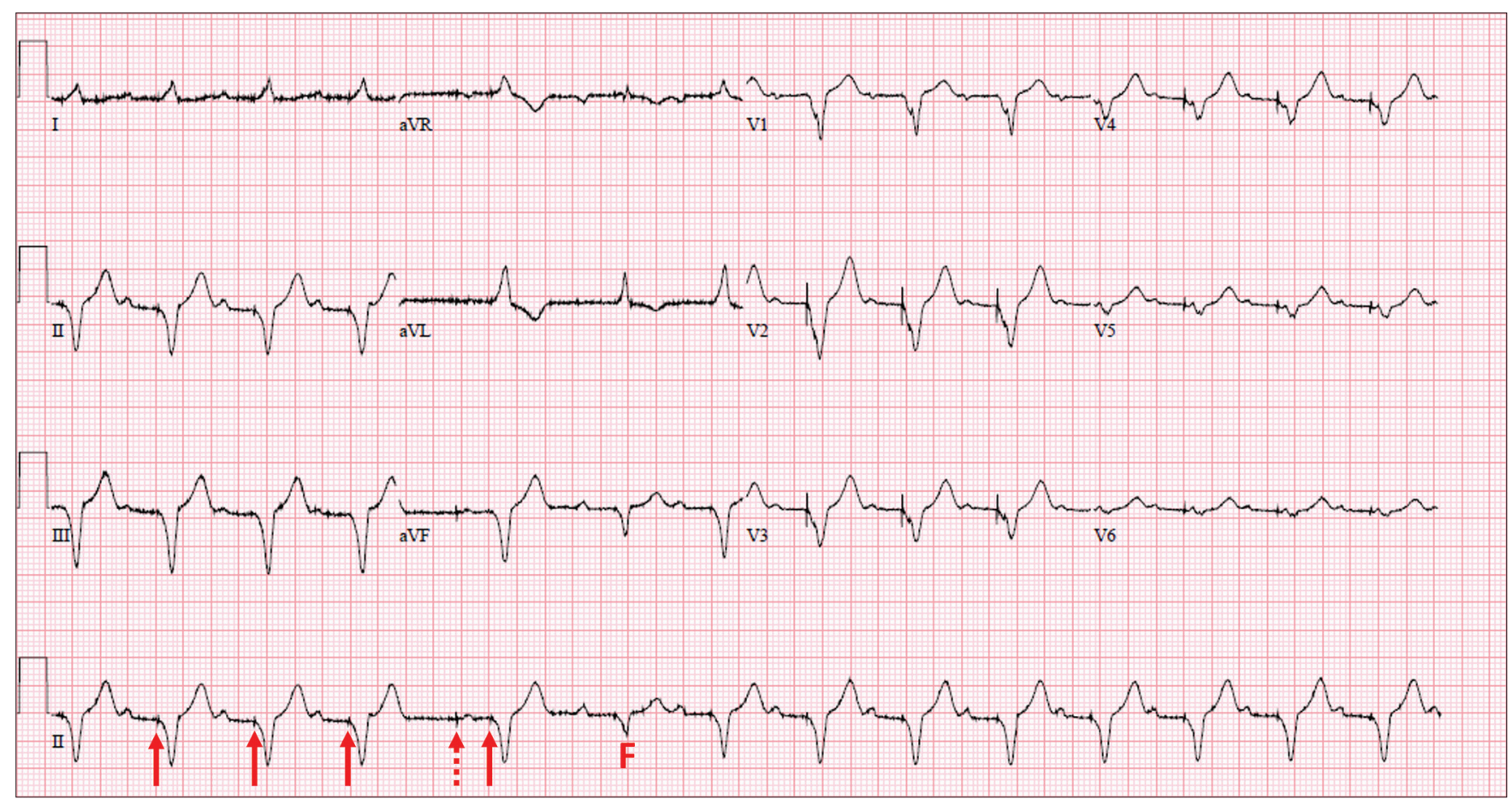

Fig. 5 Case 2: ECG initially shows intrinsic $P$ waves followed by a pacing spike (arrow) and a broad QRS complex, which is consistent with an A sense- $V$ pace response. After the fourth QRS complex, there was a sinus pause and an atrial pacing spike (dashed arrow) and a paced atrial beat, followed by a ventricular pacing spike and QRS complex (A pace-V pace response). The sixth QRS complex is a fusion beat (F) from the fusion of intrinsic QRS conduction and a paced complex. The QRS complexes on the chest leads exhibit negative concordance and are consistent with appropriate positioning of the ventricular lead at the right ventricular apex.

\section{DISCUSSION}

AV blocks are most commonly classified by severity. A first-degree AV block is defined by a prolonged P-R interval > $200 \mathrm{~ms}$ with no greater degrees of block. A second-degree AV block can be further stratified into Mobitz Type I, in which there is a gradual prolongation of the P-R interval in succeeding beats leading to a non-conducted P wave, and Mobitz Type II, in which the P-R intervals for conducted $P$ waves are the same before and after a non-conducted $\mathrm{P}$ wave. A third-degree AV block, or complete heart block, is when the atrial and ventricular beats are independent of each other (AV dissociation) due to a loss of conduction from the former to the latter. ${ }^{(1)}$ Two forms of atrioventricular block Mobitz Type I (Wenckebach phenomenon) and complete heart block with a broad infra-Hisian escape rhythm - were previously described in this journal in 2011. ${ }^{2}$

Anatomically, AV blocks can be divided into supra-, intra- or infra-Hisian. Mobitz Type I AV blocks tend to be from supra-Hisian blocks, whereas Type II AV blocks tend to be infra-Hisian. SupraHisian AV blocks are generally associated with a better prognosis, as the ensuing escape rhythm tends to be faster and more reliable. . $^{(3,4)}$ The cardiac electrical system has multiple redundancies, and the next echelon of automaticity takes over when there is a block or arrest upstream. Clues to the site of the block include the rate and morphology (width of QRS) of the escape rhythm.

Case 1 illustrates an easily missed ECG diagnosis. In a patient with $\mathrm{AF}$, the typical rhythm is one that is irregularly irregular. This is because the AV node is constantly inundated with fibrillatory waves, leading to erratic AV conductance. It is important to evaluate the underlying atrial rhythm carefully when a patient

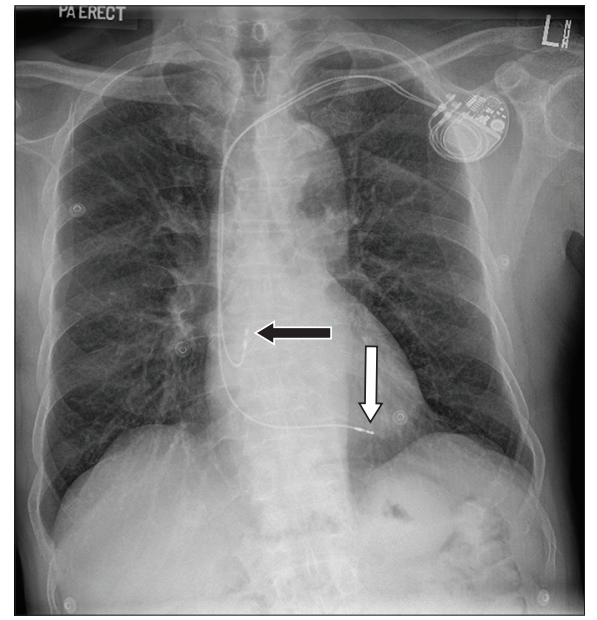

Fig. 6 Case 2: Chest radiograph following permanent pacemaker insertion shows a pulse generator implanted in the left infraclavicular area. Two leads track through the subclavian vein into the superior vena cava. The atrial lead loops upwards and anteriorly in the right atrium and is fixed in the right atrial appendage (black arrow). The ventricular lead crosses the tricuspid valve and is fixed at the right ventricular apex (white arrow).

with AF presents with regular QRS complexes. The differentials include: (a) paroxysmal atrial fibrillation, with the patient now in sinus rhythm (normal P waves preceding each QRS complex); (b) sinus arrest with junctional escapes and retrograde atrial conduction (retrograde $\mathrm{P}$ waves after each QRS complex); and (c) atrial fibrillation with complete heart block. In Case 1, the background atrial fibrillatory waves without any discrete $P$ waves confirmed that it was the third differential diagnosis.

Management of AF with complete heart block includes anticoagulation based on the patient's thromboembolic risk. ${ }^{(5)}$ 
Rate control is normally not an issue, as the patient is often intrinsically bradycardic. Permanent pacemaker implantation is indicated for complete heart block and an awake heart rate $<40 \mathrm{bpm}$.

Case 2 may also be easily missed, as the $\mathrm{P}$ wave is partially buried at the end of the T wave. This is most apparent in leads I and $\mathrm{V} 1$. In a patient who is bradycardic with a slow sinus rate, one should meticulously screen the complexes and isoelectric line between the $\mathrm{P}$ waves for any additional ones that may have been 'buried' in larger complexes. A 2:1 AV block cannot be definitively classified into Mobitz Type I or II. ${ }^{(6)}$ Clinical history and ECG can provide clues to suggest whether it is a supraHisian or infra-Hisian block. This classification is important, as the former may be managed conservatively. ECG features to note include QRS width (a wider QRS width is more likely to indicate an infra-Hisian block), a concomitant Wenckebach phenomenon on a separate rhythm strip indicating a supra-Hisian block, and the rate of escape rhythm.

Patients with an inferior ST-elevation myocardial infarction and $\mathrm{AV}$ block can often be observed for recovery of $\mathrm{AV}$ conduction after revascularisation. ${ }^{(7)}$ However, in Case 2, the left anterior descending coronary artery stenosis is more consistent with infra-Hisian blockage due to ischaemia of the interventricular septum. Additionally, the AV block persisted despite revascularisation; hence, a decision was made for permanent pacemaker implantation.

Principles of management of AV block are to first assess for grade of block and haemodynamic stability, followed by symptoms and resting heart rate. Patients should be methodically assessed for reversible acquired causes such as electrolyte imbalances, myocardial ischaemia, endocrine abnormalities, myocarditis and other inflammatory conditions of the heart, infections and drug therapy. Patients who are haemodynamically unstable due to bradycardia should be treated as per Advanced Cardiac Life Support protocols, including temporary venous cardiac pacing. Intravenous chronotropes such as atropine, isoprenaline or dopamine may be utilised in specific situations to maintain adequate cardiac output. ${ }^{(8,9)}$ All reversible causes of AV block should be addressed. In patients with irreversible causes of AV block, indications for permanent pacemaker insertion include symptomatic bradycardia, periods of asystole $\geq 3$ seconds, infra-Hisian escape rhythms, escape rhythms $<40$ bpm and complete heart block. ${ }^{(1)}$

ABSTRACT Atrioventricular (AV) block is an AV conduction disorder that can manifest in various settings, with varying symptomaticity and severity. The electrocardiogram is a key diagnostic tool for management, and careful interpretation is necessary to institute the correct management. We described two cases of patients with bradycardia due to AV blocks and discussed the electrocardiogram interpretation and management.

Keywords: atrioventricular conduction block, atrioventricular dissociation, electrocardiography, heart block

\section{REFERENCES}

1. Epstein AE, Dimarco JP, Ellenbogen KA, et al; American College of Cardiology; American Heart Association Task Force on Practice Guidelines; American Association for Thoracic Surgery; Society of Thoracic Surgeons. ACC/AHA/HRS 2008 Guidelines for device-based therapy of cardiac rhythm abnormalities. Heart Rhythm 2008; 5:e1-62.

2. Yeo TJ, Teo SG, Soo WM, Poh KK. Variations of atrioventricular block. Singapore Med J 2011; 52:330-4.

3. Dhingra RC, Denes P, Wu D, Chuquimia R, Rosen KM. The significance of second degree atrioventricular block and bundle branch block. Observations regarding site and type of block. Circulation 1974; 49:638-46.

4. Strasberg B, Amat-Y-Leon F, Dhingra RC, et al. Natural history of chronic second-degree atrioventricular nodal block. Circulation 1981; 63:1043-9.

5. January CT, Wann LS, Alpert JS, et al; American College of Cardiology/American Heart Association Task Force on Practice Guidelines. 2014 AHA/ACC/HRS guideline for the management of patients with atrial fibrillation: a report of the American College of Cardiology/American Heart Association Task Force on Practice Guidelines and the Heart Rhythm Society. J Am Coll Cardiol 2014; 64:e1-76.

6. Barold SS, Hayes DL. Second-degree atrioventricular block: a reappraisal. Mayo Clin Proc 2001; 76:44-57.

7. Behar S, Zissman E, Zion M, et al. Prognostic significance of second-degree atrioventricular block in inferior wall acute myocardial infarction. SPRINT Study Group. Am J Cardiol 1993; 72:831-4

8. Neumar RW, Otto CW, Link MS, et al. Part 8: adult advanced cardiovascular life support: 2010 American Heart Association Guidelines for Cardiopulmonary Resuscitation and Emergency Cardiovascular Care. Circulation 2010; 122(18 Suppl 3):S729-67.

9. Ching CK, Leong SH, Chua SJ, et al; National Resuscitation Council Singapore. Advanced Cardiac Life Support: 2016 Singapore Guidelines. Singapore Med J $2017 ; 58: 360-72$ 


\section{SINGAPORE MEDICAL COUNCIL CATEGORY 3B CME PROGRAMME} (Code SMJ 201807A)

Question 1. The following are forms of atrioventricular blocks:

(a) First-degree heart block.

(b) Mobitz Type I (Wenckebach) second-degree heart block.

(c) Sick sinus syndrome.

(d) Complete heart block.

Question 2. The following statements describe atrioventricular blocks accurately:

(a) First-degree heart block is defined as a prolonged R-R interval > 200 ms.

(b) Mobitz Type I (Wenckebach) second-degree heart block is characterised by fixed P-R intervals before and after a non-conducted $\mathrm{P}$ wave.

(c) Atrioventricular dissociation is a feature of complete heart block.

(d) Complete heart block cannot be present if the heart rate is $>40$ beats per minute.

Question 3. The following electrocardiography (ECG) findings are more suggestive of a supra-Hisian atrioventricular block:
(a) Narrow QRS complexes.
(b) Evidence of supra-Hisian block on other ECGs.
(c) Right bundle branch morphology.
(d) Wide QRS complex.

Question 4. The following are indications for permanent pacemaker implantation:

(a) Asymptomatic first-degree heart block.

(b) Asymptomatic complete heart block with heart rate $<40$ beats per minute.

(c) Symptomatic supra-Hisian atrioventricular block.

(d) Asymptomatic Mobitz Type II second-degree heart block with a wide QRS complex.

Question 5. Advanced Cardiac Life Suppport protocols for management of patients with unstable bradycardia include:
(a) Intravenous atropine.
(b) Valsalva manoeuvre.
(c) Transcutaneous pacing.
(d) Addressing reversible causes.

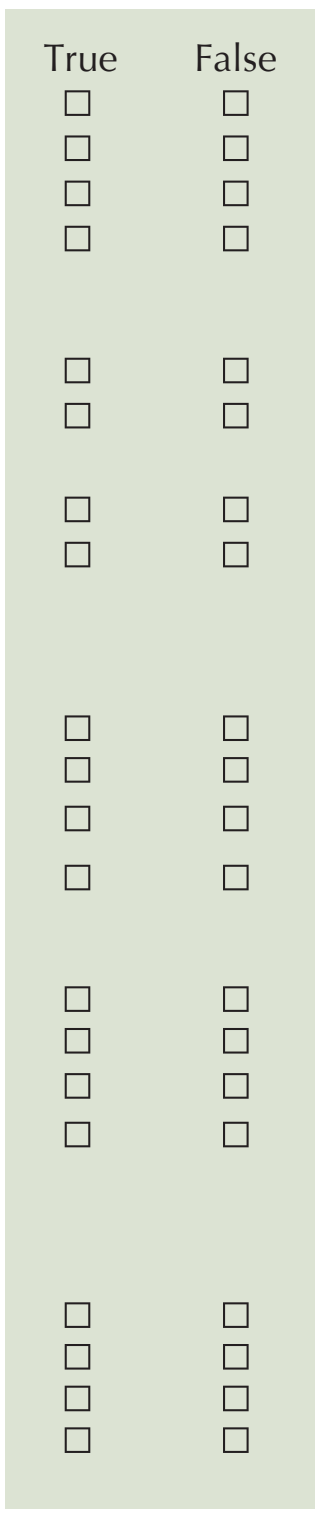

\section{Doctor's particulars:}

Name in full:

MCR no.:

Specialty:

Email:

\section{SUBMISSION INSTRUCTIONS:}

Visit the SMJ website: http://www.smj.org.sg/current-issue and select the appropriate quiz. You will be redirected to the SMA login page.

For SMA member: (1) Log in with your username and password (if you do not know your password, please click on 'Forgot your password?'). (2) Select your answers for each quiz and click 'Submit'.

For non-SMA member: (1) Create an SMJ CME account, or log in with your SMJ CME username and password (for returning users). (2) Make payment of SGD 21.40 (inclusive of $7 \%$ GST) via PayPal to access this month's quizzes. (3) Select your answers for each quiz and click 'Submit'.

\section{RESULTS:}

(1) Answers will be published online in the SMJ September 2018 issue. (2) The MCR numbers of successful candidates will be posted online at the SMJ website by 5 September 2018. (3) Passing mark is $60 \%$. No mark will be deducted for incorrect answers. (4) The SMJ editorial office will submit the list of successful candidates to the Singapore Medical Council. (5) One CME point is awarded for successful candidates. (6) SMC credits CME points according to the month of publication of the CME article (i.e. points awarded for a quiz published in the December 2017 issue will be credited for the month of December 2017, even if the deadline is in January 2018).

Deadline for submission: (July 2018 SMJ 3B CME programme): 12 noon, 29 August 2018. 